\title{
A PROPERTY OF CERTAIN FUNCTIONS WHOSE STURMIAN DEVELOPMENTS DO NOT TERMINATE *
}

BY O. D. KELLOGG

Let $\left[u_{k}(x)\right]$ be a set of continuous Sturmian functions defined on the interval $(a, b)$, i.e., solutions of an equation of the form

$$
\frac{d}{d x}\left[k(x) \frac{d}{d x} u(x)\right]+\lambda g(x) u(x)=0,
$$

each satisfying two linear homogeneous self-adjoint boundary conditions and corresponding to a value of $\lambda$ for which this is possible. We assume that the coefficients of the differential equation have derivatives of all orders, and that $g(x)$ does not vanish in the closed interval $(a, b)$, nor $k(x)$ on the open interval. Let $f(x)$ denote a function with derivatives of all orders, satisfying boundary conditions to be specified presently. We proceed to call attention to a property which such functions must have if their developments in series of the functions $u_{k}(x)$ are not to terminate.

We denote by $a_{k}$ the $k$ th generalized Fourier coefficient of $f$ :

$$
a_{k}=\int f u_{k} g d x,
$$

where we have omitted argument $x$, and limits of integration, $a$ and $b$. No ambiguity will result from the abbreviation. If in the integral, we replace $u_{k} g$ by its value obtained from the differential equation (1), and integrate by parts, we obtain

$$
a_{k}=-\frac{1}{\lambda_{k}} \int\left(k f^{\prime}\right)^{\prime} u_{k} d x
$$

where the integrated terms have been omitted on the assumption that $f$ satisfies the same self-adjoint boundary conditions as the $u_{k}$. Under this assumption, they vanish. We define a series of functions as follows:

$$
f_{n}=-\frac{\left(k f_{n-1}^{\prime}\right)^{\prime}}{g}, \quad f_{0}=f .
$$

\footnotetext{
* Presented to the Society, Feb. 25, 1922.
} 
We now subject $f$ to the hypothesis that it, and these derived functions, all satisfy the same boundary conditions as the $u_{k}$. It then appears that for all $n$,

$$
a_{k}=\frac{1}{\lambda_{k}{ }^{n}} \int f_{n} u_{k} g d x .
$$

This is a simple generalization of the long familiar equation for the coefficients of a Fourier series. It is rather in the inference drawn from it, than in the generalization itself, that the interest lies. From the equation (5) we pass to an inequality. Let $B_{k}$ denote the maximum of $\left|(b-a) g(x) u_{k}(x)\right|$, and $F_{n}$ the maximum of $\left|f_{n}(x)\right|$. Then, evidently,

$$
\left|a_{k}\right| \leqq B_{k} F_{n} /\left|\lambda_{k}\right|^{n}, \quad \text { or } \quad F_{n} \geqq\left(\left|a_{k}\right| / B_{k}\right)\left|\lambda_{k}\right|^{n} \text {. }
$$

It follows that unless the development of $f(x)$ terminates, $F_{n}$ must, for all $n$ greater than a determinable number $N$, exceed any exponential function of $n, A e^{p n}$, because of the known property of the characteristic numbers $\lambda_{k}$ of having infinity as the only limit point of their absolute values.

In other words, if $F_{n}$ is less than any such exponential function, for positive $A$ and $p$ and for infinitely many values of $n$, $f(x)$ is a homogeneous linear function of the $u_{k}(x)$ with constant coefficients.

This property takes on special interest when $k$ and $g$ are constant, for in this case the $f_{n}$ are proportional to derivatives of $f$. With suitable boundary conditions, it then takes the form: if the periodic function $f(x)$ has derivatives of all orders, it is either a trigonometric polynomial, or else the maximum of the absolute value of its nth derivative exceeds any exponential function $A e^{p n}$ for all $n$ from a certain one on.*

In the case of analytic functions of a complex variable, we have the result:

Either $f(z)$ is a polynomial, or else the maximum of the absolute value of its nth derivative on any circle lying entirely within its domain of analyticity exceeds any exponential function $A e^{p n}$ from a given $n$ on.

HaRVARD UNIVERSITY

* The result in this particular form was announced to the Society Dec. 2, 1911. See this Bulletin, vol. 18, p. 234. 